Z Rheumatol 2009 · 68:529-529

DOI 10.1007/s00393-009-0447-1

Online publiziert: 20. August 2009

(c) Springer Medizin Verlag 2009

\section{Redaktion}

W. Graninger, Graz

W. Graninger

Klinische Abteilung für Rheumatologie, Medizinische Universität Graz

\title{
Evidenz für die Anwendung von Physik am menschlichen Körper?
}

Liebe Kolleginnen und Kollegen,

elektrischer Strom, Temperaturänderungen und Bewegung werden seit langer Zeit als Heilmittel, besonders in der muskuloskelettalen Medizin, angewendet. In unserer Zeit der Kostenbewusstheit können diese Behandlungsformen kritisch hinterfragt werden, da nur $\mathrm{z}$. T. medizinstatistisch und medizinökonomisch ausreichende Rechtfertigungen für die Kostenträger vorliegen.

****

37. Kongress der Deutschen Gesellschaft für Rheumatologie e.V. (DGRh)

23. Jahrestagung der Assoziation für orthopädische Rheumatologie e.V.

(ARO)

19. Jahrestagung der Gesellschaft für Kinder- und Jugendrheumatologie (GKJR)

***

Liebe Leserinnen, liebe Leser,

die Abstracts zum 37. Kongress der DGRh erscheinen in diesem Jahr online als Supplement 1 der Zeitschrift für Rheumatologie. Sie finden diese im Volltextarchiv der Zeitschrift unter:

www.ZeitschriftfuerRheumatologie.de Mitglieder der DGRh haben zudem die Möglichkeit, aus dem geschlossenen Mitgliederbereich (unter www.dgrh.de) direkt auf das Volltextarchiv zuzugreifen.

Ihre Redaktion

\section{(7) Physikalische Benhandlungsformen werden aus Kostengründen kritisch hinterfragt}

Die Fachärztinnen für Physikalische Medizin können nicht immer aus Studien schöpfen, welche die Kriterien der evidenzbasierten Medizin erfüllen, und auch mancher Rheumatologe ist erstaunt, wenn z. B. in den rezenten britischen Empfehlungen zum Management der rheumatoiden Arthritis die physikalische Therapie lediglich als Bewegungsprophylaxe bei Osteoporose vorkommt.

Dennoch werden auch in der nahen Zukunft aus Empirie Patienten mit schmerzhaften Affektionen des Bewegungsapparates durch therapeutisches mechanisches Training und Bewegungstherapie, Thermotherapie und Elektrotherapie dankbar zu einer Schmerzreduktion kommen.
$\mathrm{Zu}$ einigen Aspekten der vorhandenen Evidenz aber erhalten Sie in den folgenden Artikeln anschauliche Informationen. Sie sollen auch den Betreibern und Verfechtern bisher nicht gesichert wirksamer Methoden Appetit machen, wissenschaftliche Validierungen anzustreben. Die Selbstzahler könnten sonst besonders bei chronisch rheumatischen Erkrankungen - weniger werden.

Mit den besten Empfehlungen

Ihr

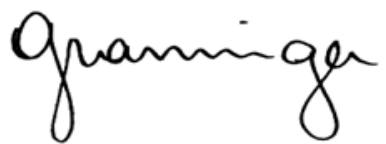

W. Graninger

\section{Korrespondenzadresse}

Prof. Dr. W. Graninger

Klinische Abteilung für Rheumatologie,

Medizinische Universität Graz

Auenbruggerplatz 15, $8036 \mathrm{Graz}$, Österreich winfried.graninger@klinikum-graz.at 